\title{
Miras and Galactic Populations
}

\author{
M.O. Mennessier \\ GRAAL, Univ Montpellier II/CNRS, F-34095 Montpellier
}

X. Luri, F. Figueras, J. Torra

Departament d'Astronomia i Meteorologia, Univ Barcelona, Espagne

\begin{abstract}
In order to exploit the imminent HIPPARCOS data we have developed a new Maximum-Likelihood method to determine luminosity calibrations and kinematical properties with as bias-free as possible estimates. In most cases the samples are inhomogeneous, containing stars of several types. The method includes a way to separate the different groups. We applied the method to Mira variables contained in the HIPPARCOS Input Catalogue.
\end{abstract}

\section{Introduction}

In our Maximum-Likelihood method to calibrate luminosity, the modeling of the samples has been made as realistic as possible - including the effects of the observational constraints, inhomogeneities of the sample, galactic rotation, observational errors and interstellar absorption - so the results are as bias-free as possible (Luri et al., 1993).

\section{Sample}

The sample is composed of 90 Mira variables contained in the HIPPARCOS Input Catalogue with known proper motions and radial velocities. Mean apparent magnitudes are from 75 years AAVSO data (H. Boughaleb, 1994). We take into account the process of selection used to decide the Miras to be included in the HIPPARCOS Input Catalogue - see ESA SP-111, Vol. II - basically affecting the distribution of apparent magnitudes. Selection effects in proper motions and radial velocities are not considered important after comparison with the distributions of Miras in the GCVS.

\section{Results}

Using Wilks test on likelihood, the separation of three groups of stars is decided. By the Maximum Likelihood method we get the estimates shown in Table 1. Each star can be assigned to a group. So we obtain an independent estimation of the distance of each star from observations at maximum or min- 
imum luminosity. The agreement between both these estimates is good. The results from observations at minimum luminosity seem more reliable and agree with the estimates of velocities in the galactic plane and motion perpendicular to the galactic plane.

\section{Conclusion}

The Miras in our sample can be considered as belonging to three populations: thick disk (group 1); old disk stars (group 2) with the same velocities, aligned with the Sun: they could belong to a stream; possible halo stars (group 3), but the small value of $\sigma_{M}$ is probably not real (too small number of stars). However, all stars in group 3 have small period and early spectral type and seem to be very luminous. This agrees with Jura \& Kleiman (1992) results and with Whitelock's review paper (these proceedings).

Table 1. Estimates of mean intrinsic $V$ luminosity, mean velocity $(\mathrm{km} / \mathrm{s})$ and galactic disk height $(\mathrm{pc})$ and percentage of stars in each group

\begin{tabular}{|c|c|c|c|c|c|c|c|c|c|c|c|c|}
\hline & \multicolumn{2}{|c|}{ group 1} & \multicolumn{2}{|c|}{ group 2 } & \multicolumn{2}{|c|}{ group 3} & \multicolumn{2}{|c|}{ group 1} & \multicolumn{2}{|c|}{ groun 2 } & \multicolumn{2}{|c|}{ group 3} \\
\hline$\overline{M_{0}}$ & 0.4 & 0.6 & -1.8 & 0.9 & -2.5 & $\overline{0.4}$ & 6.3 & 03 & $=24$ & $\overline{08}$ & $=0.1$ & 08 \\
\hline $\begin{array}{l}M_{0} \\
\sigma_{M}\end{array}$ & $\begin{array}{l}0.4 \\
1.0\end{array}$ & 0.2 & $\begin{array}{l}-1.0 \\
10\end{array}$ & 0.4 & 0.1 & 0.4 & $\begin{array}{l}0.0 \\
1.4\end{array}$ & 0.1 & 0.7 & $\begin{array}{l}0.0 \\
0.4\end{array}$ & 0.1 & $\begin{array}{l}0.0 \\
0.6\end{array}$ \\
\hline$U_{0}$ & -16 & 7 & -49 & 10 & -69 & 28 & -19 & 9 & -51 & 8 & -65 & 70 \\
\hline$\sigma_{U}$ & 44 & 5 & 12 & 6 & 85 & 25 & 46 & 5 & 12 & 6 & 86 & 60 \\
\hline$V_{0}$ & -33 & 6 & -37 & 5 & -139 & 55 & -38 & 8 & -39 & 4 & -220 & 60 \\
\hline$\sigma_{V}$ & 39 & 6 & 0 & 2 & 83 & 29 & 41 & 3 & 0 & 5 & 93 & 50 \\
\hline$W_{0}$ & -5 & 6 & -22 & 12 & -9 & 32 & -7 & 3 & -23 & 9 & -4 & 160 \\
\hline$\sigma_{W}$ & 23 & 7 & 12 & 8 & 205 & 39 & 31 & 2 & 16 & 7 & 300 & 130 \\
\hline$Z_{0}$ & 329 & 81 & 47 & 18 & 390 & 180 & 900 & 800 & 51 & 25 & 550 & 260 \\
\hline$\%$ & 75 & 8 & 9 & 2 & 16 & 7 & 84 & 3 & 10 & 2 & 6 & 3 \\
\hline & & $\operatorname{arent}$ & magnit & e at & naxim & & & aren & aggnit & $\overline{d e} a$ & minimu & \\
\hline
\end{tabular}

\section{References}

Boughaleb, H. 1994, these Univ. Montpellier II

Jura, M. 1992, ApJS, 79, 105

Luri, X., Mennessier, M.O., Figueras, F., \& Torra, J. 1994, in "New approaches in Classification and Data Analysis", Springer-Verlag, p.672 